\title{
Mapping microbes on the move
}

A low-cost imaging strategy gives scientists the 'big picture' on locomotive behavior of microorganisms in solution.

Bacterial cultures are busy places, and characterizing the underlying parameters of cellular movement can be like trying to understand a city's traffic laws from 30,000 feet above. Cellular motility is typically studied via laborious tracking experiments, wherein many swimming bacteria are imaged individually so that researchers can build a statistically strong model of their movement. However, a research team led by Wilson Poon and postdoctoral researcher Vincent Martinez of the University of Edinburgh has shown that an optical technique developed by materials scientists could enable faster and more accurate analyses.

In differential dynamic microscopy (DDM), the point is not to generate highresolution renderings of individual bacteria but rather to obtain a lower-resolution panorama with numerous cells. This means that virtually any light microscope can be used to collect data. "You can zoom out a lot and have a very large field of view that contains up to 10,000 cells, which gives you very robust statistics," explains Martinez. Tracking is still required initially to determine the types of motility a microbe engages in-for example, Escherichia coli bacteria both propulsively 'run' and passively tumble. By coupling tracking-based models with Fourier transform analysis of low-resolution imaging data obtained over a time course of a few minutes, DDM can quickly chart the dynamic behavior of cultures of swimming cells. "We perform basic tracking to obtain trajectories; thereafter, we can perform DDM for high-throughput motility characterization and don't need to come back to tracking anymore," says Martinez.

The researchers used DDM to study E. coli as well as the algae Chlamydomonas reinhardtii, which moves via an entirely different mechanism. For both organisms, DDM quickly generated accurate movement data from tens of thousands of cells within a three-dimensional volume. By comparison, most single-cell tracking is performed in two dimensions because of the equipment and computational costs associated with highresolution three-dimensional imaging.

The researchers are now adapting DDM to track directed cellular movement in denser cultures and in response to environmental cues, and Martinez envisions diverse potential applications ranging from sperm motility assays to characterization of how pathogens invade the viscous mucosa lining the mammalian gastrointestinal tract.

\section{Michael Eisenstein}

RESEARCH PAPERS

Martinez, V.A. et al. Differential dynamic microscopy: a high-throughput method for characterizing the motility of microorganisms. Biophys. J. 103, 16371647 (2012). 